\title{
Bohm's Super Implicate Order and the Quantum Zeno Effect
}

\author{
Marcus Schmieke* \\ * Head of Scientific Research, Institute of Existential Consciousness Research (ECR), Berlin, Germany \\ *Adjunct Professor, Dev Sanskriti University, Haridwar, India \\ https://doi.org/10.36018/dsiij.v19i.241
}

\begin{abstract}
David Bohm extended his model of quantum mechanics to include super implicate orders that can be iterated an infinite number of times. With each implication step, the measure of wholeness increases, which is introduced here as an additional dimension $\mathrm{x}_{5}$. The sum total of the time as well as the energy intervals of the single steps should be finite, from which a quantum Zeno effect can be derived. This could lead to the fact that the objects of higher-order levels, like collective psychic, archetypal and spiritual contents as well as mathematics, logic, and natural laws are strongly slowed down in their evolution.
\end{abstract}

Keywords. David Bohm, Super Implicate Order, Zeno Effect, Consciousness, Copenhagen Interpretation, Quantum Potential, Burkhard Heim

\section{BOHM'S QUANTUM POTENTIAL AND THE IMPLICATE ORDER}

In the last 20 years, Bohm's quantum mechanics has gained renewed attention in expert circles because it represents a realistic alternative to the Copenhagen interpretation of quantum theory and has been confirmed many times by applications in specific fields, opening new perspectives (1).

Bohm's account of quantum mechanics replaces the complementarity of particles and waves propagated by Bohr and Heisenberg, with the simultaneous coexistence of an explicit order consisting of the physical particles and an implicate order consisting of a wave function. The wave function acts on a particle by means of a quantum potential which results from the second spatial derivative of the wave function:

$$
Q=-\frac{\hbar^{2}}{2 m} \frac{\nabla^{2} R}{R}
$$

Writing the wave function using the two real parameters $\mathrm{R}$ and $\mathrm{S}$ with

$\Psi(\mathbf{r} ; \mathrm{t})=R e^{\frac{\mathrm{iS}(\mathbf{r} ; \mathrm{t})}{\hbar}}$

we arrive at the Schrödinger equation:

$\frac{\partial S}{\partial t}+\frac{(\nabla S)^{2}}{2 m}+V+Q=0$

While $V$ represents the external potential, the quantum potential $Q$ thus results as internal energy added to the kinetic energy of the particle, which is derived from the second derivative of the real part of the wave function and goes to zero if Planck's quantum of action is set to zero as in the classical approximation. 
In this representation, the localized form of the particle with defined momentum can be retained, while the holistic quantum effect of the entire environment of the particle is mediated by the quantum potential Q.
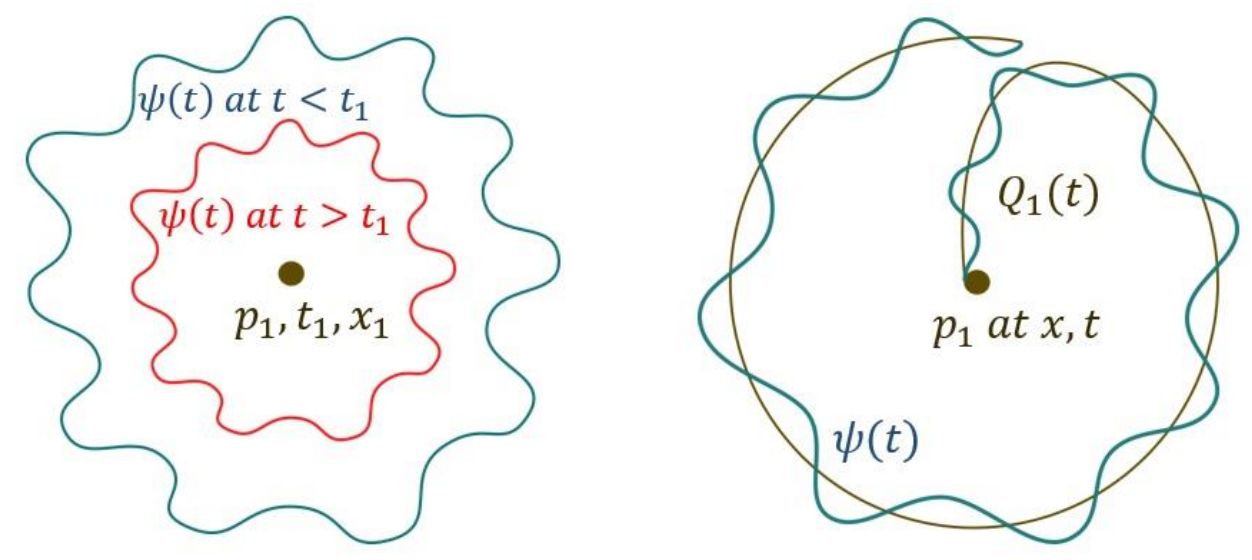

Figure 1. Nils Bohr's versus David Bohm's Interpretation of Quantum Mechanics

In a very simplified form, the left figure symbolizes the representation of a measurement of a particle $\mathrm{p}_{1}$ at time $\mathrm{t}_{1}$. Before the measurement, the particle is represented only by a wave function, which collapses at time $t_{1}$ due to the interaction with a classic measuring device, and then evolves again as a wave function. Outside the time $t_{1}$, at which the particle was found at the place $\mathrm{x}_{1}$, only a probability is given with the help of Born's rule to find the particle $\mathrm{p}_{1}$ at a certain place:

$P\left(x_{1}\right)=\left|\left\langle x_{1} \mid \Psi_{S}(t)\right\rangle\right|^{2}$

In this representation, the interaction with the measuring device has been neglected, which is limited to the time $t_{1}$. On the right side of figure 1 , the same facts are shown from the perspective of Bohm's quantum mechanics. Here, the particle $\mathrm{p}_{1}$ always has a well-defined location $\mathbf{x}_{1}$ and momentum $\mathbf{P}_{1}$, and at the same time, the wave function exists at every instant. The particle $p_{1}$ is guided by the quantum potential $\mathrm{Q}$ at each instant, which converts the information contained in the
The following figure illustrates the difference between the Copenhagen interpretation of quantum mechanics and the Bohmian model: wave function into a guiding energy Q. The physical process of measurement is treated in Bohmian quantum mechanics in the same way as any other interaction of the particle with its environment (2).

In the right graph of Figure 1, the quantum potential Q conveys the effect of the whole (wave function) on the part (the particle). The information represented by the wave function is activated by the exact parameters of the particle so that it can act on the particle through the quantum potential Q. Neither particle nor wave function contains the complete information, but only the combination of both. The wave function is physically relevant information activated by the exact position of the particle. It contains not only the effect of the environment on the particle with certain spatiotemporal coordinates but that of all other possibilities. Only the presence of the particle $p_{1}$ at time $t_{1}$ at location $\mathbf{x}_{1}$ activates this information in the form of the quantum potential Q. Since also in the Bohmian representation of quantum mechanics the Heisenberg uncertainty 
relation can be derived and is valid (3), the exact coordinates of $\mathbf{x}_{\mathbf{1}}$ and $\mathbf{p}_{\mathbf{1}}$ are never exactly known to the observer here either, so that Born's rule continues to describe the probability of certain measurement results. In contrast to the Copenhagen interpretation of Bohr and Heisenberg, in the Bohmian representation, the physical properties of the particle are real and physically effective at any time.

David Bohm has called the wave function active information, which contains as an implicate order the holistic non-local influences of the whole on the part/the particle. The quantum potential mediates the influence of the whole on the part and thus represents the meaning context of the particle. The image of the wave function, quantum potential, and particle sketched by Bohm includes the essential quantum properties of the system such as non-locality, acausality, and probability interpretation, but in contrast to the Copenhagen interpretation, it is strictly deterministic. If, in the Bohmian account, the wave function as well as the position and momentum of a particle or system were given at a certain time, then the complete temporal evolution of the system would be fixed at that time. Ontologically speaking, the future would be already determined. However, it cannot be known by an observer, because the Heisenberg uncertainty principle prevents exact measurement and thus the knowledge of all parameters. In principle, however, this image of reality does not allow causal or acausal effects of a non-physical aspect of reality on matter, as represented for example by the consciousness or the psyche of a human being.

\section{CONSCIOUSNESS AND THE SUPER IMPLICATE ORDER}

Bohm himself, however, was convinced of a parallel existence of matter and consciousness and also attributed causative possibilities to consciousness itself. This could be one of the main reasons why Bohm continued developing his model and proposed a repetition of the process represented by the formation of a quantum potential and thus another implicate order.

“... one could begin by supposing, for example, that as the quantum potential constitutes active information that can give form to the movements of the particles, so there is a super-quantum potential that can give form to the unfoldment and development of this first-order quantum potential. This latter would no longer satisfy the laws of the current quantum theory, which latter would then be an approximation, working only when the action of the super-quantum potential can be neglected. Of course, there is no reason to stop here. One could go on to suppose a series of orders of super-quantum potentials, with each order constituting information that gives form to the activity of the next lower order (which is less subtle)" (4).

Bohm seeing the higher levels of the series of implicate orders primarily in relation to consciousness is evident in the following quotation from an interview given in 1989:

"I have an idea of an implicate order and beyond that a super-implicate order, and so on to orders that are more and more subtle. I say there are many more subtle levels. The word 'subtle' has a root sub-text meaning 'finely woven'. You may think of nets of consciousness that are finer and finer, or we may think of capturing finer and finer aspects of the implicate order. This could go on indefinitely" (5).

Bohm sees in the higher levels of the series more complex structures of the external material reality and more comprehensive forms of consciousness. Thus, an axis reaching into the depth of the 
iterations results, which measures a higher complexity, more comprehensive wholeness and higher form of consciousness of the described explicate order with increasing iterations. A development of material complexity and consciousness are interacting here, because more complex material systems are to be expected above all in the biological area, which already in their simplest forms are accompanied by consciousness. For higher biological life forms, further developing psychological structures and dynamics are resulting, independently of their biological basis, which leads up to cultural and spiritual connections. Such an axis Phi was already proposed by Pylkkänen (6) on the basis of the Integrated Information Theory by Tononi (7). The information referred to as Phi measures the degree of connectedness of integration, calculated from the ratio of the total information of the system to the sum of the information of its parts.

\section{THE $\mathrm{X}_{\mathbf{5}}$-AXIS FOR MEASURING WHOLENESS AND COMPLEXITY}

A similar parameter $\mathrm{x}_{5}$ measuring the wholeness and complexity of a system was already found by the German physicist Burkhard Heim (8), based on mathematical necessities that arose in the generalization of Einstein's theory of gravity. Together with another $\mathrm{x}_{6}$-axis, these two dimensions form an information space which controls particles in $\mathrm{R}^{4}$ via the time axis by means of energetic-informational processes. Burkhard Heim suggested dividing the full range of the $\mathrm{x}_{5}{ }^{-}$ axis into four sections referring to qualitatively different realms of reality, which in this paper are introduced as physis, bios, psyche and logos (Heim called the fourth level pneuma):

\section{Physis}

This first section on the $\mathrm{x}_{5}$-axis covers the range from subquantum level structures, elementary particles up to macromolecules like the DNA.
Each level is a new wholeness, integrating all the parts from the lower levels, like a molecule integrates atoms and an atom integrates elementary particles like electrons, protons and neutrons. The DNA itself is the last level of integration belonging to physis, as it already manifests new properties like reproduction and self-referential processes.

\section{Bios}

With the DNA molecule, matter already reaches a complexity that enables a multitude of processes underlying the life process. This includes, for example, the construction of RNA molecules, which in turn enable protein synthesis. Furthermore, the DNA molecule is able to divide itself and thus produce identical copies of itself. The information carried by DNA is the basis for the differentiation of life. Errors during replication can cause mutations which are responsible for the further development of life over longer periods of time. Just at this point, by controlling from a level of higher wholeness (higher $\mathrm{x}_{5}$ value), integral information can be streaming in, which can control the evolution of life. For this reason the DNA molecule as the last stage of material already forms the first stage of living. The most important characteristic, which constitutes life, is the ability to store information, to translate it into material structures, to connect the information with environmental influences and to process, change or develop information accordingly. On higher levels of life, such as the cell, these properties are even more pronounced. The cell is a clearly limited, self-organizing, information-processing system, possessing in addition one of the most important characteristics of life, namely self-referentiality. The Bios level is structured by many levels of implications.

The DNA is a part of the nucleus, which in turn is a part of the cell. The cell is a living being that 
may exist for itself. At the beginning of the evolution of life there was the unicellular organism, still existing today. In the course of billions of years, cells have organized themselves in the form of organisms, these in turn consisting of billions of cells. All these single cells do not follow only their own purpose of existence anymore, but interact under the control of a higher whole, of the organism. All these levels of life can be understood as different levels of wholeness, each of them containing all the information of its parts plus the integral information of its own purpose and existence.

On each level of higher wholeness, non-local and acausal effects like quantum entanglements of its components are a part of the integral information which corresponds to the difference of $x_{5}$ values of the levels. The gradation of life leads up to the level of the biosphere, in which the most diverse forms of life interact with each other and in turn form a whole. No living being can exist independently of other living beings and therefore the overall coordination of the biosphere is also life-sustaining from this level of wholeness. In the communication of living beings among themselves phenomena of social behavior appear on this level, which suggests inner psychic processes.

\section{Psyche}

The higher forms of life have developed, in the form of their central nervous system, probably the most complex information-processing system in the universe. As a result of this system, the complex neuronal processes and networks have given rise to corresponding inner contents and experiences which are generally referred to as the psyche. Therefore, from the point of view of the approach discussed herein, it is obvious to assume that behind the complex neuronal activities there is a further gradation of implicate orders, which enable a psychological influence on neurobiological processes.

Mental activities have a fundamentally different quality from biological life processes, since they are of an inner experiential quality that can only be inadequately objectivized. They are reflection by essence, which acquires self-referentiality by further reflection on the reflection process itself, which corresponds to self-consciousness (9). Within the psyche, every horizon of consciousness possesses an unconscious background, so that also within the conscious psyche higher implicate orders are to be expected.

\section{Logos}

The human psyche makes use of mental forms (in the sense of the German term geistig or the Greek logos) of perception that have developed over thousands of years of cultural history. These are concepts, words, language, forms, ideas, depthpsychological archetypes, but also mental concepts that go far beyond the psyche, such as logic, mathematics and religion. All these mental contents have arisen only in the collective cultural history and go far beyond the development of a single individual. Even the laws of nature expressed in terms of mathematics on the basis of logic can be regarded as developed forms which are founded in an iteration of implicate orders reaching into infinite depth (10). The infinite depth of the spiritual, which leads to the conception of timelessness and eternity, can be seen as an indication that the infinite series of implicit orders could converge in the infinite in timelessness. This is where time comes into play.

\section{INFINITE SERIES OF IMPLICATE ORDERS}

Bohm himself describes the series of unfolding and enfolding of an explicate order into an implicate order as a movement that unfolds from implicate depth toward manifest reality, 
transforming energy at each step. It begins in the implicate depth of consciousness and manifests in the exterior as physiological processes of the body. Likewise, energy can flow in the opposite direction, so that the meaning of external events in the form of perceptions enters into consciousness:

"From each level of somatic unfoldment of meaning, there is ... a further movement leading to activity on to a yet more manifestly somatic level, until the action finally emerges as a physical movement of the body that affects the environment. So one can say that there is a twoway movement of energy, in which each level of significance acts on the next more manifestly somatic level and so on, while perception carries the meaning of the action back in the other direction" (11).

This sequence of implicate orders must be infinite, because every finite series would end with a deepest level $\mathrm{m}$, which would completely determine all other levels as the last implicate order. Thereby reality and with it also consciousness would be just as determined as in classical physics. Consciousness and the human mind, whose wholeness corresponds to the deeper levels of the sequence would have no causal influence on the events, but would only be represented as passive levels by this model. Thus, Bohm's model would have fallen behind the Copenhagen interpretation, which at least leaves an option for non-causal influences by the real random process of the collapse of the wave function.

Each step $\mathrm{n}$ of this movement transforms a certain amount of energy $E_{n}$ and takes a certain period of time $t_{n}$. In order to allow back-forward directed movements of energy and information along the $\mathrm{x}_{5}$-axis, the total sum of the transformed energy $E=\sum_{n=1}^{\infty} E_{n}$ and the total time period $t=$ $\sum_{n=1}^{\infty} t_{n}$ need to be finite. On the $\mathrm{x}_{5}$-axis the series has to converge towards a finite value of $\mathrm{x}_{5}$ in order to define the process within limited time, energy and momentum. Each step of this unlimited converging series on the $\mathrm{x}_{5}$-axis according the last quote from David Bohm corresponds to an unfoldment of meaning from a implicate information resulting in activities on a more manifest explicate level, which again becomes the implicate order of another more manifest explicate level.

In this way, a single step of this series can be seen as an observation of reality described by the series of implicate orders behind it. This is valid both for the movement considered from the inside to the outside, in which a change on deeper levels spreads out in the direction of manifested physical reality, and the direction considered from the outside to the inside, in which starting from a measurement in the manifested area an activation of implicate orders behind it can occur up to infinity.

The infinite convergent series of super implicate orders can therefore be seen as an equally infinite series of observations taking place between the different levels of the series. What is observed is the underlying reality manifesting itself in the form of the converging series. The special status of observations in the Copenhagen interpretation of quantum theory is based on the fact that an observation is a reflection which maps different levels onto each other. However, the reflection follows a different logic than that which is objectified by the reflection (12). If reflections are iterated as in this case, a multilevel depth of selfconsciousness arises from it, which is reflected in an increasing wholeness of the objectified manifested reality. The entire process of the infinite series of reflections in turn faces the transcendent self-consciousness in a final 
reflection, the subject of an ultimately complete reality (13). This experienced and lived reflection faces as its implicit consciousness the iterative holomovement of reality, encompassing both matter and the empirical consciousness interwoven in it, described by Bohm, making it possible in the first place.
The repeated observation of a quantum state slows down its temporal evolution according to the temporal density of observation. This process, known as the quantum Zeno effect (14), leads to a slowing down of the development of the corresponding layer of the underlying reality with increasing $\mathrm{n}$ and thus deeper iteration steps of the implicate orders.

\section{THE QUANTUM ZENO EFFECT IN THE DEPTHS OF ITERATION}



Figure 2. Infinite iteration of the implicate orders and the total reflection as the underlying implicate consciousness

The quantum Zeno effect would particularly increase the temporal stability of the deeper level $\mathrm{x}_{5}$-structures, corresponding to the psyche or the logos. Applying such a Zeno effect in quantum neurology Henry Stapp suggested that the influence of the psyche on neuronal quantum processes could be achieved by slowing them down due to repeated observation through the consciousness of the psyche resulting (15).

Furthermore the fourth level, which I call logos, might evolve extremely slow under the Zeno effect. This refers to forms of logos-like mathematical formulas, laws of nature, aesthetical form, psychological archetypes etc. Here, Lee
Smolin's idea of the evolution of the laws of nature as precedent cases might be of interest (16). On the deeper levels of psyche and logos, the involved energies become smaller and smaller, but their influence simultaneously becomes larger. On the deeper levels, the influences of quantum entanglement with parallel infinite series overcome the silos of individual experiences and emerge as a participation in a collective unconscious (psyche) and a collective medium of meaning (logos, geistig, spiritual). The infinity of depth of the reflections corresponds to the infinity of widths of being as visible in Figure 2. 


\section{A SECOND ZENO EFFECT SUPPRESSES THE DEVELOPMENT OF THE QUANTUM POTENTIAL}

A different scenario of a quantum Zeno effect within the physical level of the implicate order seems to be increasing von Neumann entropy and decoherence if applied locally to the brain, which would contradict the statement of the Zeno effect stabilizing the mental states. In an article on Henry Stapp's approach to the problem of consciousness, Georgiev (17) concludes that the quantum Zeno effect must therefore refer not only to the local quantum state of the brain, but to the global state of the brain entangled with its environment. Especially for mental (psyche) and beyond that, collective mental states (logos), exactly such non-local quantum relations should be considered to represent a more complex wholeness. This is exactly the conclusion to which the Bohmian model is leading. Georgiev deduced in the same article that such entangled states are actually slowed down and stabilized by the quantum Zeno effect.

Hiley and deGosson (2018) (18) discussed the influence of the Zeno effect on the Bohmian quantum potential. Their calculations have shown that when a particle moves in a way that leaves a continuous trace in observed reality, such as an alpha particle passing through an ionization chamber, the quantum potential of the particle cannot evolve in time fast enough to exert a significant influence in its equation of motion. This Zeno effect leads to a classical trajectory of the particle and quantum effects resulting from the quantum potential can be neglected.

On the higher levels of super implicate orders, this can mean that continuous observation or monitoring of a process leads to it behaving according to the logic of classical physics, i.e. two-valued logic, while processes that are not continuously observed can exhibit quantum phenomena of an acausal and non-local nature. De Gosson (19) calls an object that follows the Bohm equation of motion a metatron. In the series of super implicate orders, organisms (bios), thoughts (psyche) and ideas (logos) can be considered as such characteristic metatrons. Thus a thought may be an object of a constant observation, which would lead to the fact that it develops in mental causality and locality. Synchronistic (20) (acausal) and mentally entangled (non-local) interactions with other mental contents would then not be expected. A thought which, left to itself, slips into the unconscious and only later spontaneously comes back into consciousness to be observed again, could in the meantime, by the quantum potential which can no longer be neglected, become entangled with thoughts of other persons or be influenced by contents of the collective psyche. Quantum effects of the psyche could fertilize its development, enrich it with feelings or deepen it by intuitions. Inside the observation of consciousness, psychological contents would follow the two-valued logic, while outside they could evolve in a poly-contextural environment not subject to two-valued logic (21).

\section{ENERGY DISTRIBUTIONS ON THE $\mathrm{X}_{5^{-}}$ AXIS}

Fig. 3 below shows the distribution of the amounts of energy transformed per each iteration step of such a series. Here it is considered that the sum of all energies $E=\sum_{n=1}^{\infty} E_{n}$ is finite and also the sequence of the $\mathrm{x}_{5}$ values of the iterated implicate orders converges with $\mathrm{n}$ to infinity.

$x_{5}^{\infty}=\lim _{n \rightarrow \infty} x_{5}(n)$

This assumption is confirmed by the derivation of the $\mathrm{x}_{5}$ parameters by Burkhard Heim (22). However, the limit $x_{5}$ of the sequence may shift in time on the $\mathrm{x}_{5}$ axis as the described system changes its complexity. 


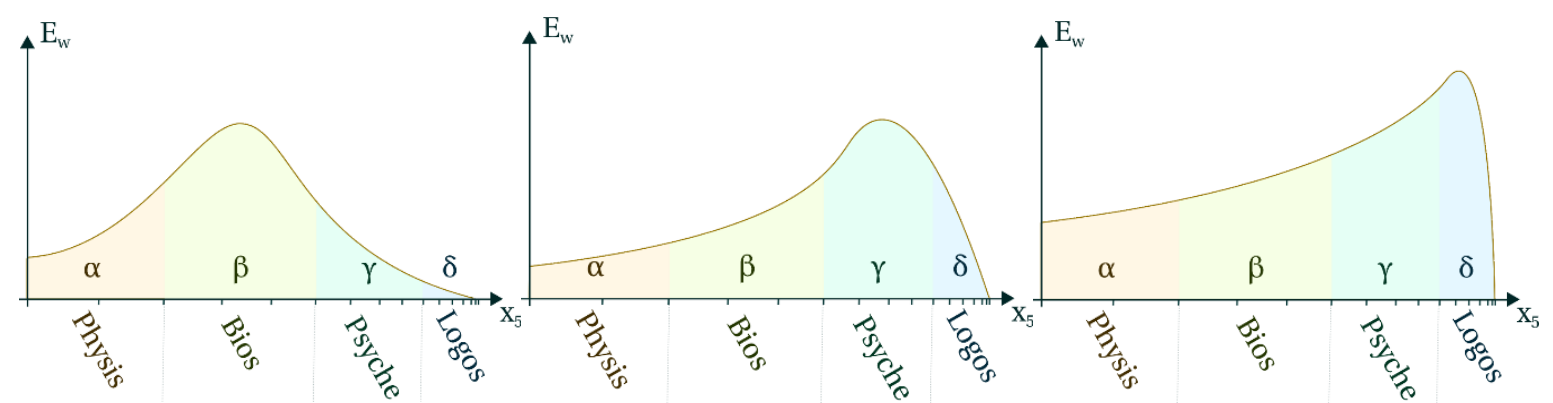

Figure 3. Energy Spectra on the $x_{5}$-axis with focus on Bios (life process), psyche (thoughts) and Logos (mathematical principles or ideas)

The graphs in Figure 3 represent the energy distributions of the quantum potentials over the different levels of the super implicit orders. The first plot shows a life process where the maximum of the energy distribution is in the bios domain. The second illustration shows a psychic process and the third a process of ideas, the maxima of each again lie in the associated domains. Although the sum of the energy potentials to the right of the respective maximum is small, there exists for every arbitrarily deep representation or experience level an implicate order lying behind it with a non-zero contribution in the associated quantum potentials of transformed energy. Thus, from any point of view, the leftward explicit process is determined once the position of all explicate metatrons is known, while the infinite contribution of implicate orders to the right of it means that the process always remains indeterminate from a deeper point of view. Changes in a more holistic background can alter reality and keep the process open to influences exerted by the whole. The sum total of the super implicate orders can be defined as a dynamic information field (23), complementing the explicate physical reality. Its nature is active information as meaning.

\section{CONCLUSION}

The Zeno effect may be involved in determining events at two levels in Bohm's model of super implicate orders. The first level relates to the question how, at a certain level of explicitness, the quasi-classical behavior of the metatron comes about. By a Zeno effect the evolution of the quantum potential is slowed down so much, according to Hiley and de Gosson, that a classical motion arises if the process is continuously monitored. Transferred to deeper levels of the implicate order this allows conclusions about the behavior of thoughts and ideas in their motion between the conscious and unconscious as well as between the collective and the individual.

With the unfolding of the implicate order from the infinite depth of the iteration, as well as with the enfolding of explicate realizations into the infinity of the implicate, a Zeno effect may be responsible for the fact that collective psychic as well as ideal contents change only very slowly or not at all, up to mathematical, logical or natural laws. In the realm of depth, time seems to stand still.

\section{REFERENCES}

1. Benseny A, Albareda G, Sanz ÁS, Mompart J, Oriols X. Applied Bohmian mechanics. Eur Phys J D [Internet]. 2014 Oct;68(10). Available from: http://dx.doi.org/10.1140/epjd/e2014-50222-4

2. Bohm D, Hiley BJ. Measurement understood through the quantum potential approach. Found Phys [Internet]. 1984 Mar;14(3):255-74. Available from: http://dx.doi.org/10.1007/BF00730211 
3. Bohm D, Hiley BJ. The Undivided Universe: An Ontological Interpretation of Quantum Theory (London: Routledge, 1993); chapter 6.4 The meaning of the uncertainty principle p. 114-116

4. Bohm, D. (1990) Unfolding the Implicate Order, Excerpts from interview with David Bohm by Louwrien Wijers, 1989 in Art Meets Science and Spirituality in a Changing Economy, SDU publishers, Amsterdam

5. Bohm, D. (1990) Unfolding the Implicate Order, Excerpts from interview with David Bohm by Louwrien Wijers, 1989 in Art Meets Science and Spirituality in a Changing Economy, SDU publishers, Amsterdam

6. Pylkkänen P. Is there Room in Quantum Ontology for a Genuine Causal Role for Consciousness? In: The Palgrave Handbook of Quantum Models in Social Science [Internet]. Palgrave Macmillan UK; 2017. p. 293-317. Available from: http://dx.doi.org/10.1057/978-1-137-49276-0 14

7. Tononi G, Boly M, Massimini M, Koch C. Integrated information theory: from consciousness to its physical substrate. Nat Rev Neurosci [Internet]. 2016 May 26;17(7):450-61. Available from: http://dx.doi.org/10.1038/nrn.2016.44

8. Illobrand Von Ludwiger, T. Auerbach, Heim's Theory of Elementary Particle Structures, Journal of Scientific Exploration. Vol. 6, No. 3, pp. 217-231, 1992 0892-33 10192 O 1992 Society for Scientific Exploration

9. Marcus Schmieke, Orthogonal Complementarity, Tattva Viveka 2019, www.acadedemia.edu.

10. Unger RM, Smolin L. The Singular Universe and the Reality of Time [Internet]. Cambridge University Press; 2014. Available from: http://dx.doi.org/10.1017/CBO9781139696487

11. Bohm, D. (2003) Soma-significance and the Activity of Meaning. In The Essential David Bohm; L. Nichol Ed., London: Routledge; pp. 158-182.

12. Gotthard Günther (1976), Cybernetic Ontology Operations, Beiträge zur Grundlegung einer operationsfähigen Dialektik, Erster Band, Felix Meiner Verlag

13. Schmieke, M. (2021). Bohm's Quantum Potential Approach to Consciousness from the Perspective of a Four-Valued Logic. Dev Sanskriti Interdisciplinary International Journal, $17, \quad 01$. https://doi.org/10.36018/dsiij.v17i.206
14. Misra B, Sudarshan ECG. The Zeno's paradox in quantum theory. Journal of Mathematical Physics [Internet]. 1977 Apr;18(4):756-63. Available from: http://dx.doi.org/10.1063/1.523304

15. Stapp, H.P. (2000a) "Decoherence, quantum Zeno effect, and the efficacy of mentaleffort."quant$\mathrm{ph} / 0003065$

16. Smolin, Lee (2012), Precedence and freedom in quantum physics, Perimeter Institute for Theoretical Physics, arXiv:arXiv:1205.3707

17. Georgiev D. Mind Efforts, Quantum Zeno Effect and Environmental Decoherence. Neuroquantology [Internet]. 2012 Sep 30;10(3). Available from: http://dx.doi.org/10.14704/nq.2012.10.3.552

18. de Gosson, Maurice,Hiley, Basil (2010), Zeno Paradox for Bohmian Trajectories: The Unfolding of the Metatron, arXiv:1010.2622

19. de Gosson MA, Hiley B. The Principles of Newtonian and Quantum Mechanics [Internet]. PUBLISHED BY IMPERIAL COLLEGE PRESS AND DISTRIBUTED BY WORLD SCIENTIFIC PUBLISHING CO.; 2001. Available from: http://dx.doi.org/10.1142/p235

20. Atmanspacher H. Psychophysical correlations, synchronicity and meaning. J Anal Psychol [Internet]. 2014 Mar 27;59(2):181-8. Available from: http://dx.doi.org/10.1111/1468-5922.12068

21. Günther G. Beiträge zur Grundlegung einer operationsfähigen Dialektik II [Internet]. Felix Meiner Verlag; $1979 . \quad$ Available from: http://dx.doi.org/10.28937/978-3-7873-2555-9

22. Illobrand Von Ludwiger, T. Auerbach, (1992) Heim's Theory of Elementary Particle Structures, Journal of Scientific Exploration. Vol. 6, No. 3, pp. 217-231

23. Schmieke, M. (2021). Quantum-Entangled Frequencies and Coherence in Bioenergetic Systems, Dev Sanskriti Interdisciplinary International Journal, 18. https://doi.org/10.36018/dsiij.v18i.226 